\title{
Hyperbaric oxygen therapy for pyoderma gangrenosum associated with ulcerative colitis
}

\author{
Hyun Il Seo, Hyun-Ju Lee, Koon Hee Han \\ Department of Internal Medicine, Gangneung Asan Hospital, University of Ulsan College of Medicine, Gangneung, Korea
}

Pyoderma gangrenosum (PG), an ulcerating skin condition, is rare in patients with ulcerative colitis (UC). We report a case of successful treatment of PG in a patient with UC using hyperbaric oxygen therapy (HBOT). The patient had UC that was in remission following treatment with mesalazine and azathioprine therapy. After visiting an orthopedic clinic, the patient opted for treatment with antibiotics and daily dressing of the ulcerative skin lesions, while azathioprine was discontinued. However, the lesions did not improve. Two months later, the patient visited a dermatologist who diagnosed the lesions as PG, and he was admitted to our unit. Surgical debridement and HBOT were performed by a plastic surgeon in the emergency department. After 3 months of HBOT and topical treatment, the patient's PG completely resolved. His UC was still in remission with mesalazine alone. HBOT may be an effective and safe alternative treatment for PG associated with UC, particularly in patients in whom anti-tumor necrosis factor agents are unnecessary. (Intest Res 2018;16:155-157)

Key Words: Pyoderma gangrenosum; Hyperbaric oxygenation; Colitis, ulcerative

\section{INTRODUCTION}

Pyoderma gangrenosum (PG) is rarely observed in patients with UC, with a prevalence of approximately $2 \%$. $^{1}$ The pathophysiology of PG is unclear. Moreover, there is no standard or specific treatment for PG. Consequently, treatment is usually based on clinician experience. In recent years, anti-tumor necrosis factor (anti-TNF) agents have been suggested as the treatment of choice for PG associated with UC. ${ }^{2-6}$ However, in some patients with UC in remission, antiTNF agents are not essential. Here, we report a case in which hyperbaric oxygen therapy (HBOT) was used to treat PG associated with UC in remission.

Received February 6, 2017. Revised April 14, 2017.

Accepted April 17, 2017. Published online July 19, 2017

Correspondence to Koon Hee Han, Department of Internal Medicine,

Gangneung Asan Hospital, University of Ulsan College of Medicine, 38

Bangdong-gil, Sacheon-myeon, Gangneung 25440, Korea. Tel: +82-33-610-

3945, Fax: +82-33-610-4960, E-mail: hankoonhee@hanmail.net

\section{CASE REPORT}

A 65-year-old man with a 17-year history of UC visited the internal medicine department of Gangneung Asan Hospital, complaining of a painful skin defect in both pretibial areas (Fig. 1A). The patient's UC was in remission because of treatment with mesalazine $1.5 \mathrm{~g}$ /day and azathioprine $50 \mathrm{mg} /$ day. The patient was also using metformin $1 \mathrm{~g} /$ day, glimepiride $3 \mathrm{mg} /$ day, and olmesartan $20 \mathrm{mg}$ /day for diabetes mellitus and hypertension. Three months earlier, he had visited a local orthopedic hospital due to the presence of ulcerative lesions in both pretibial regions. The orthopedist diagnosed the ulcerative lesions as cellulitis and prescribed antibiotics and daily dressing. The orthopedist asked the patient to discontinue azathioprine because of the unhealed lesions. The patient discussed discontinuing his use of azathioprine with his gastroenterologist. The gastroenterologist considered his medical condition as being in complete remission based on his condition during the last year, and allowed the patient to discontinue azathioprine treatment. However, the ulcerative lesions in both pretibial regions remained unhealed after 2 months, prompting the patient to visit a dermatologist. The

\footnotetext{
๑ Copyright 2018. Korean Association for the Study of Intestinal Diseases. All rights reserved.

This is an Open Access article distributed under the terms of the Creative Commons Attribution Non-Commercial License (http://creativecommons.org/licenses/by-nc/4.0)

which permits unrestricted non-commercial use, distribution, and reproduction in any medium, provided the original work is properly cited.
} 

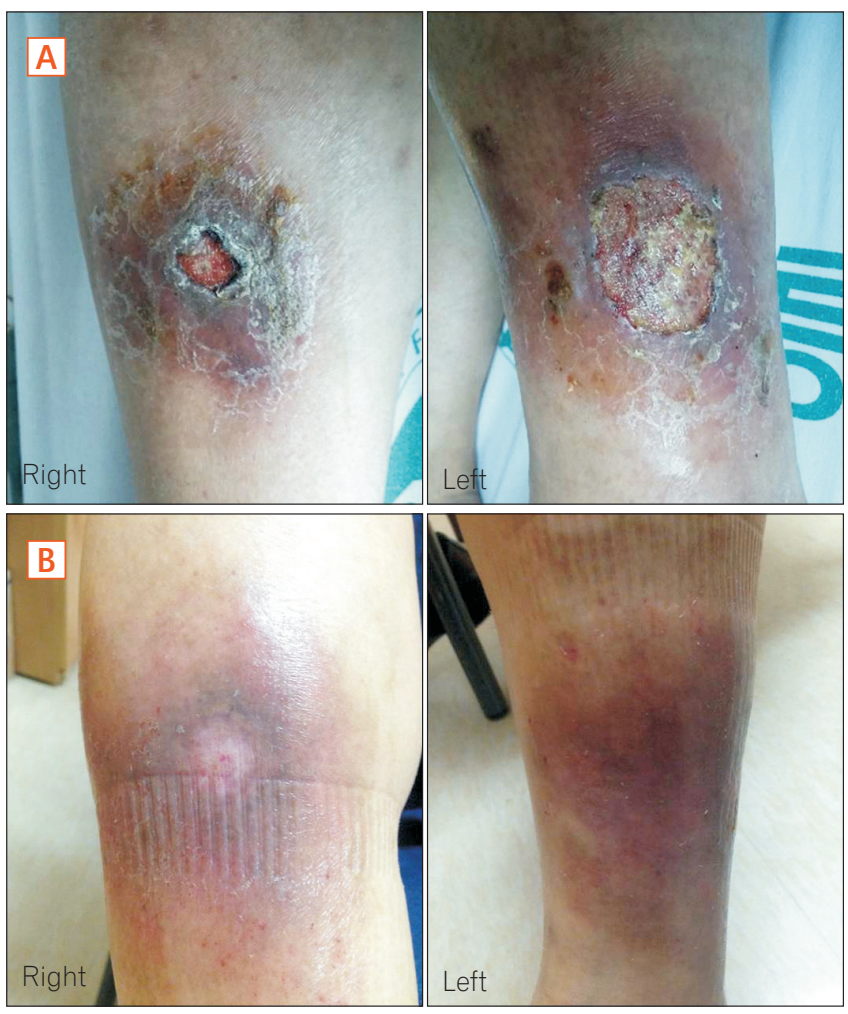

Fig. 1. (A) Photographs of both shins, showing ulcerative lesions with surrounding erythema. (B) Photographs of both shins after 3 months of hyperbaric oxygen therapy. Following ulcerative lesions resolution, only post-inflammatory hyperpigmentation remained.

dermatologist diagnosed the lesions as PG, and the patient was admitted. Upon admission, his vital signs were as follows: blood pressure, 119/64 mmHg; heart rate, 85 beats/ min; respiratory rate, 22 breaths/min; and body temperature, $36.5^{\circ} \mathrm{C}$. His laboratory data were as follows: white blood cell count, 6,200/ $/ \mathrm{mm}^{3}$; neutrophil level, $67.4 \%$; hemoglobin level, $12.0 \mathrm{~g} / \mathrm{dL}$; platelet count, $243,000 / \mathrm{mm}^{3}$; CRP level, $0.20 \mathrm{mg} /$ $\mathrm{dL}$ (normal, $<0.30 \mathrm{mg} / \mathrm{dL}$ ); and glycosylated hemoglobin level, $6.0 \%$. Lower extremity CT angiography showed no active lesion. The patient was placed on mesalazine alone for maintenance treatment of his UC. He also underwent surgical debridement of the ulcerative lesions, which was performed by a plastic surgeon, as well as HBOT for 120 minutes at 2.4 atmospheres, based on the plastic surgeon's recommendation. The patient experienced pain in the left ear while in the hyperbaric tube because his ears were not properly equalized. A ventilating (tympanostomy) tube was inserted into the left tympanic membrane and the therapy was maintained. Over the next 3 months, a total of 29 sessions of HBOT, along with topical treatment without systemic antibiotics, were administered. Subsequently, the patient's ulcerative lesions completely resolved (Fig. 1B) and his UC continues to be in remission.

\section{DISCUSSION}

PG is rare and some cases have been treated with HBOT; ${ }^{7,8}$ however, no case of PG associated with UC has received such treatment. To the best of our knowledge, this is the first case of PG associated with UC that was successfully treated with HBOT. In our case, the patient first presented to a local clinic. Because of the ulcerative lesions that were not resolved despite antibiotic therapy and daily dressing, the patient visited our dermatologist who diagnosed PG without performing a biopsy examination. PG has no pathognomonic, pathologic, or histologic findings and, occasionally, a biopsy is performed to distinguish PG from infection, malignancy, vasculitis, and insect bites. However, diagnosis of PG is based on the clinician's experience and the patient's clinical manifestations. ${ }^{9,10}$ In the present case, the anatomical location (shins) of the lesion, the findings of the dermatologist, the patient's underlying UC, a lack of abnormal lesion on CT angiography, and the clinical course supported the diagnosis of PG. Therefore, we concluded that the clinical diagnosis was PG associated with UC. Nevertheless, it is possible that this case of PG was idiopathic PG. Although up to 50\% of PG cases are idiopathic, the remaining cases seem to be associated with other conditions, with IBD being one of the most common associated diseases. ${ }^{11}$ Even though most cases of PG associated with UC develop with exacerbation of bowel disease, PG is not closely related to the activity of colitis and can develop after colectomy. ${ }^{1,12,13}$ These facts support our diagnosis. While the pathogenesis of PG is poorly understood, neutrophil dysfunction, disturbance of immune system regulation, and genetic predisposition have been proposed as possible pathogenic mechanisms. ${ }^{11}$ Many reports have revealed the use of various treatments based on clinical experience. ${ }^{9}$ Recently, anti-TNF agents have been suggested as treatment options for PG associated with UC..$^{2-4,9-11,14}$ According to previous reports, the treatment success rate is high and the response is rapid. However, it is unclear when the anti-TNF agent is to be discontinued and, although rare, adverse effects, such as respiratory infection or lymphoma, must be considered.

Use of HBOT for wound healing is well known. HBOT is performed in a closed hyperbaric chamber with $100 \%$ oxygen at 2 to 3 atmospheres. HBOT improves the local hypoxia of wounds by increasing the amount of oxygen dissolved in the blood. HBOT has some bactericidal effects, inhibits 
vasoconstriction, and improves angiogenesis. These actions may enhance wound healing and tissue regeneration. In general, the duration of a single treatment varies from 45 to 120 minutes at 2 to 3 atmospheres. Although some adverse effects may occur with HBOT, such as reversible myopia, claustrophobia, and equalization problems due to pressure differences in either or both ears, most are reversible or selflimiting. In general, HBOT is considered to be a very safe modality when used according to standard protocols. ${ }^{15}$

Cases of PG treated with HBOT have been previously reported. However, to the best of our knowledge, this is the first report of PG associated with UC that was treated with HBOT. Thus, this is the first case to highlight the possibility of HBOT as a safe and effective treatment choice for PG associated with UC, especially in patients who do not require anti-TNF agents. Moreover, HBOT precludes any concern about the adverse effects of anti-TNF agents.

\section{FINANCIAL SUPPORT}

The authors received no financial support for the research, authorship, and/or publication of this article.

\section{CONFLICT OF INTEREST}

No potential conflict of interest relevant to this article was reported.

\section{AUTHOR CONTRIBUTION}

H.I.S. contributed to main write up of the case report; H.J.L. helped data and reference selection; K.H.H. planned the case report and patient enrollment; all the authors reviewed and approved the final version to be published.

\section{REFERENCES}

1. Callen JP. Pyoderma gangrenosum. Lancet 1998;351:581-585.

2. Juillerat P, Christen-Zäch S, Troillet FX, Gallot-Lavallée S, Pannizzon RG, Michetti P. Infliximab for the treatment of disseminated pyoderma gangrenosum associated with ulcerative colitis: case report and literature review. Dermatology 2007; 215:245-251.
3. Brooklyn TN, Dunnill MG, Shetty A, et al. Infliximab for the treatment of pyoderma gangrenosum: a randomised, double blind, placebo controlled trial. Gut 2006;55:505-509.

4. Arivarasan K, Bhardwaj V, Sud S, Sachdeva S, Puri AS. Biologics for the treatment of pyoderma gangrenosum in ulcerative colitis. Intest Res 2016;14:365-368.

5. Regueiro M, Valentine J, Plevy S, Fleisher MR, Lichtenstein GR. Infliximab for treatment of pyoderma gangrenosum associated with inflammatory bowel disease. Am J Gastroenterol 2003;98:1821-1826.

6. Argóelles-Arias F, Castro-Laria L, Lobatón T, et al. Characteristics and treatment of pyoderma gangrenosum in inflammatory bowel disease. Dig Dis Sci 2013;58:2949-2954.

7. Vieira WA, Barbosa LR, Martin LM. Hyperbaric oxygen therapy as an adjuvant treatment for pyoderma gangrenosum. An Bras Dermatol 2011;86:1193-1196.

8. Altunay I, Kucukunal A, Sarikaya S, Tukenmez Demirci G. A favourable response to surgical intervention and hyperbaric oxygen therapy in pyoderma gangrenosum. Int Wound J 2014;11:350-353.

9. Ruocco E, Sangiuliano S, Gravina AG, Miranda A, Nicoletti G. Pyoderma gangrenosum: an updated review. J Eur Acad Dermatol Venereol 2009;23:1008-1017.

10. Harbord M, Annese V, Vavricka SR, et al. The first European evidence-based consensus on extra-intestinal manifestations in inflammatory bowel disease. J Crohns Colitis 2016;10:239-254.

11. Braswell SF, Kostopoulos TC, Ortega-Loayza AG. Pathophysiology of pyoderma gangrenosum (PG): an updated review. J Am Acad Dermatol 2015;73:691-698.

12. Holmlund DE, Wählby L. Pyoderma gangrenosum after colectomy for inflammatory bowel disease: case report. Acta Chir Scand 1987;153:73-74.

13. Cox NH, Peebles-Brown DA, MacKie RM. Pyoderma gangrenosum occurring 10 years after proctocolectomy for ulcerative colitis. Br J Hosp Med 1986;36:363.

14. Meng X, Zhu X, Chen L, Wu J, Liu G, Xia B. Pyoderma gangrenosum of articulations carpi associated with ulcerative colitis: one case report. Int J Clin Exp Med 2015;8:19184-19187.

15. Tibbles PM, Edelsberg JS. Hyperbaric-oxygen therapy. N Engl J Med 1996;334:1642-1648. 